\title{
On the phase structure of lattice QCD with twisted-mass Wilson fermions at non-zero temperature
}

The tmfT Collaboration: *

M. Müller-Preussker ${ }^{\dagger}$

Humboldt-Universität zu Berlin, Institut für Physik, D-12489 Berlin, Germany

E-mail: mmp@physik.hu-berlin.de

\section{Ernst-Michael Ilgenfritz}

Humboldt-Universität zu Berlin, Institut für Physik, D-12489 Berlin, Germany

Universität Heidelberg, Institut für Theoretische Physik, D-69120 Heidelberg, Germany

E-mail: ilgenfriephysik.hu-berlin.de

\section{Karl Jansen *}

NIC, DESY, D-15738 Zeuthen, Germany

E-mail: Karl.Jansen@desy.de

\section{Maria Paola Lombardo}

Laboratori Nazionali di Frascati, INFN, I-100044 Frascati, Roma, Italy

E-mail: Mariapaola.Lombardo@lnf.infn.it

\section{Owe Philipsen and Lars Zeidlewicz}

Universität Münster, Institut für Theoretische Physik, D-48149 Münster, Germany,

E-mail: O.Philipsen@uni-muenster.de

E-mail: Zeidlewicz@uni-muenster.de

Malik Kirchner, Marcus Petschlies, David Schulze, and Carsten Urbach

Humboldt-Universität zu Berlin, Institut für Physik, D-12489 Berlin, Germany

E-mail: Malik.Kirchner@physik.hu-berlin.de

E-mail: Marcus.Petschlies@physik.hu-berlin.de

E-mail: David.Schulze@physik.hu-berlin.de

E-mail: Carsten.Urbach@physik.hu-berlin.de

In this talk we give an overview of the $3 \mathrm{D}$ phase diagram of two-flavour non-zero temperature lattice QCD with twisted-mass Wilson fermions and a tree-level Symanzik-improved gauge action. We present a first feasibility study at maximal twist and, for the quenched case, we demonstrate automatic $\mathscr{O}(a)$-improvement to work.

The XXVII International Symposium on Lattice Field Theory

July 26-31, 2009

Peking University, Beijing, China

\footnotetext{
*Supported by DFG via SFB/TR9 "Computational Particle Physics"

$\dagger$ Speaker.
} 


\section{Introduction}

The aim of this work was to explore the applicability of the twisted-mass Wilson fermion formulation [1,2] as described in the review by A. Shindler [3] for investigations of lattice QCD at non-zero temperature. The use of the staggered-fermion formulation has computational advantages [4], but remains conceptually controversial [5]. On the other hand, the often used cloverimproved Wilson fermion formulation requires to determine action and operator specific improvement coefficients. The twisted-mass formulation, combined with a tree-level Symanzik-improved gauge action $[6,7]$, appears to be a challenging alternative for non-zero temperature lattice simulations, since it offers automatic $\mathscr{O}(a)$-improvement by tuning the bare quark mass parameter only. It allows high-statistics simulations in the range of pion masses $m_{\pi} \gtrsim 270 \mathrm{MeV}$.

As a first step we had to characterize the phase structure of the model by locating the transition/crossover lines and surfaces in the three-dimensional $\beta-\kappa-\mu_{0}$-space. The results of this study supporting a conjecture for the phase diagram by M. Creutz from the chiral perturbation theory point of view $[8,9]$ were already presented in Refs. [10, 11]. Here we give an overview of the phase diagram but concentrating on the thermal transition surface. Moreover, we discuss a first feasibility study carried out at maximal twist for large quark mass. In the quenched case we are going to demonstrate that automatic $\mathscr{O}(a)$ improvement also works in the finite-temperature case.

\section{The 3D phase diagram and the thermal transition}

The $\beta-\kappa$-phase diagram for two-flavour lattice QCD with clover-improved Wilson fermions has been thoroughly studied for small time-extent $N_{\tau}=4,6$ a few years ago by the CP-PACS collaboration $[12,13]$. A schematic view of the emerging phase structure is shown in the left panel of Fig. 1. The cusp of the strong coupling Aoki phase (see $[14,15]$ and references therein) - the latter (in the infinite-volume limit) characterized by a non-vanishing expectation value $\left\langle\bar{\psi} i \gamma_{5} \tau^{3} \psi\right\rangle$ indicating the spontaneous breakdown of a combined parity-flavour symmetry - seemed tightly connected with the thermal transition line $\kappa_{t}(\beta)$.

Here, we consider Wilson fermions with the additional twisted mass term $\mu_{0} \bar{\psi} i \gamma_{5} \tau^{3} \psi$. For the gauge action the tree-level Symanzik-improved gauge action is employed. The emergence of the twisted mass parameter $\mu_{0}$ requires to study a more complicated 3D phase diagram. For lattice sizes $N_{\tau}=8, N_{\sigma}=16$, we were able to show [11] that the Aoki phase ends somewhere inside the interval $\beta=3.0, \ldots, 3.4$ and, around $\beta=3.4$, becomes replaced by a region of metastabilities indicating a first order transition area (the shaded area in the right panel of Fig. 1), a remnant of a transition known also in the zero-temperature case [16, 17, 18, 19].

In what follows we are concentrating on the thermal transition seen at values $\beta \gtrsim 3.65$ and not too small $\mu_{0}$ (otherwise we are still running into the metastability region). Since the hopping parameter $\kappa$ and the twisted mass parameter $\mu_{0}$ are directly connected with the bare quark mass

$$
m_{q}=\sqrt{\frac{1}{4}\left(\frac{1}{\kappa}-\frac{1}{\kappa_{c}}\right)^{2}+\mu_{0}^{2}},
$$

we expect a cone-like structure of surfaces of equal physics around the critical chiral line $\kappa=$ $\kappa_{c}(\beta), \mu_{0}=0$. As a first step one can scan the phase diagram in a larger $\kappa$-range in order to see 

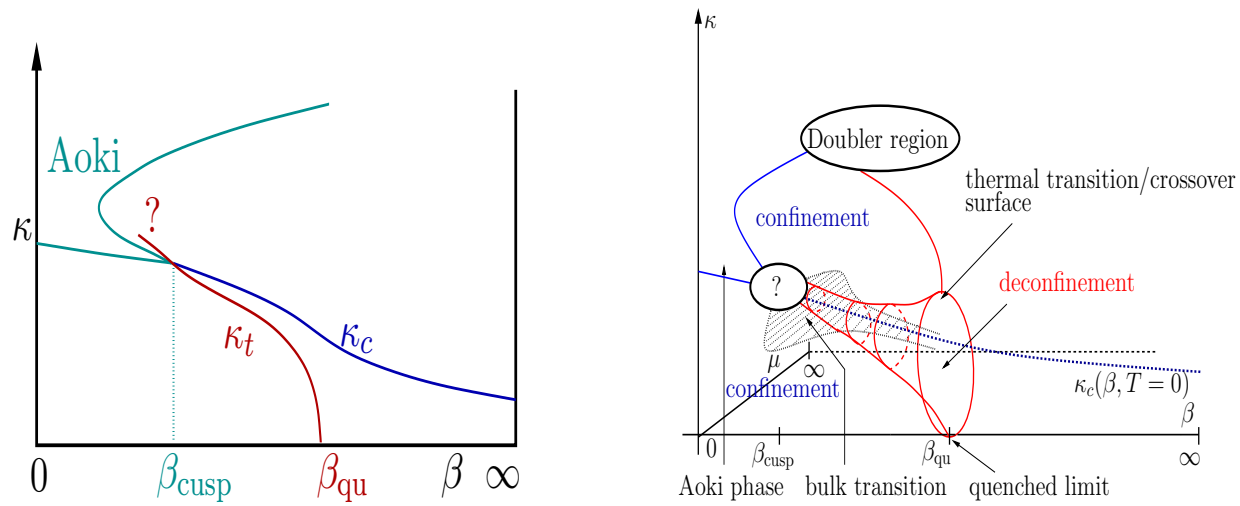

Figure 1: Schematic view of the phase structure as seen in older investigations [13] for a temporal lattice extent $N_{\tau}=4,6$ (left) and as found in this work [11] with twisted-mass fermions in the $\beta-\kappa-\mu_{0}-$ diagram for $N_{\tau}=8$ (right).

how the thermal transition surface extends above $\kappa_{c}(\beta)$. The result is shown in Figs. 2. For $\beta$ values $\beta=3.4,3.45,3.65,3.75$ from the steep rises of the Polyakov loop and from maxima of its susceptibility we observe very clear signals for a thermal transition in $\kappa$. But additionally, for $(\beta=$ $\left.3.75, \mu_{0}=0.005\right)$, we see a tiny $\kappa$-interval around $\kappa_{c}=0.166$ where the Polyakov loop exhibits a comparably little maximum, which could have been easily overlooked. Thus, with rising $\kappa$ starting from values below $\kappa_{c}$ we pass through subsequent confinement-deconfinement, deconfinementconfinement transitions (or better crossovers) below and above $\kappa_{c}$, respectively, followed again by a confinement-deconfinement transition far above $\kappa_{c}$. By additional $\beta$-scans at fixed $\kappa>\kappa_{c}$ we convinced ourselves that the two transitions above $\kappa_{c}$ in fact create a unique surface opening up at larger $\beta$-values and reaching the fermion doubler areas at much higher $\kappa$. This confirms the cone shape structure of the thermal transition as predicted by Creutz [9].
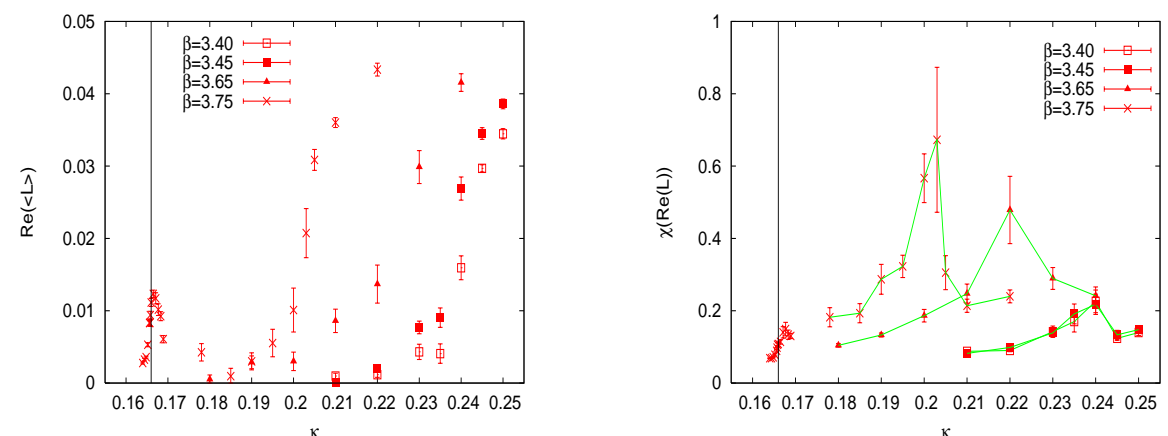

Figure 2: $\kappa$-scans of the Polyakov loop (left) and Polyakov loop susceptibility (right) for various $\beta$-values $\left(\beta=3.4,3.45,3.65\right.$ for $\mu_{0}=0.0068 ; \beta=3.75$ for $\left.\mu_{0}=0.005\right)$. Vertical lines mark $\kappa_{c}(\beta=3.75)$.

Zooming into the region around $\kappa_{c}(\beta)$ the Polyakov loop and its susceptibility behave as shown in Figs. 3. The maxima or shoulders of the Polyakov loop susceptibility shown in the right panel indicate smooth transitions or crossovers. For $\beta=3.75, \mu_{0}=0.005$ this can be clearly 

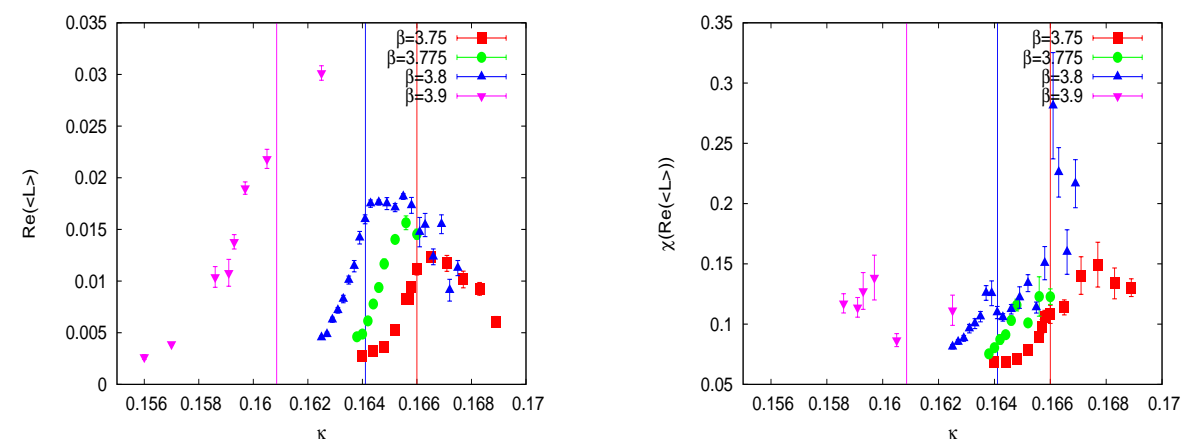

Figure 3: Zoom into the behaviour of the Polyakov loop (left) and its susceptibility (right) versus $\kappa$ for various $\beta$ and $\mu_{0}=0.005$. Vertical lines from left to right mark the chiral critical values $\kappa_{c}(\beta)$ for $\beta=$ $3.9,3.8$ and 3.75 , respectively.

seen in Fig. 4, where also the so-called pion norm has been considered. The Gaussian shape lines are fitted to enlighten the position of the expected crossover. Note that at the given $\beta$ and $\kappa_{c}(\beta)=0.166$ the value $\mu_{0}=0.005$ can be related to a pion mass value $m_{\pi} \simeq 400 \mathrm{MeV}$ and a temperature $T \simeq 210 \mathrm{MeV}$. This is close to values recently reported by the DIK collaboration [20].
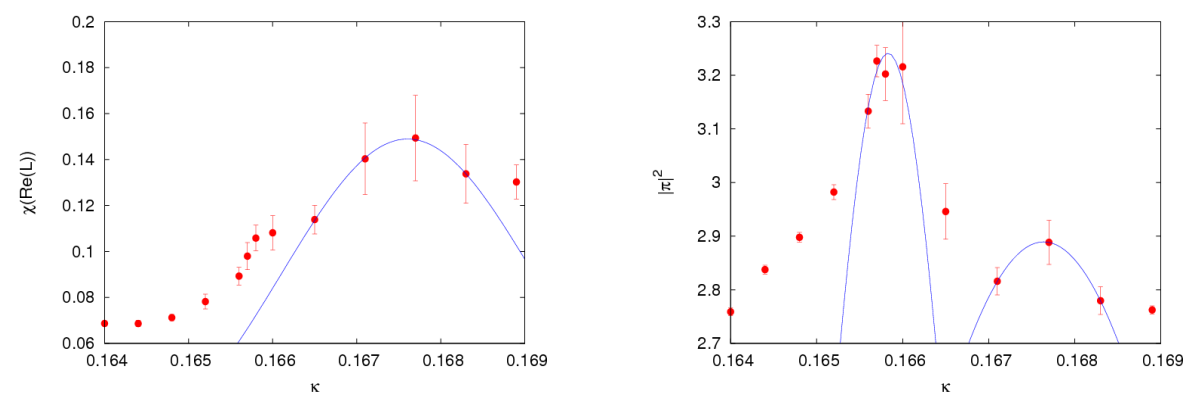

Figure 4: Polyakov loop susceptibility (left) and pion norm (right) versus $\kappa$ both for $\beta=3.75$ and $\mu_{0}=$ 0.005 .

We tried to figure out how far the crossover or transition cone extends in the $\mu_{0}$-direction. Although we collected $O\left(10^{4}\right) \mathrm{HMC}$ trajectories per point this turned out to be a quite difficult task, because of the weak and noisy signals seen in the plaquette and Polyakov loop susceptibilities, in the corresponding autocorrelation times as well as in the pion norm variable. From $\kappa$-scans for the Polyakov loop at $\beta=3.75$ and various $\mu_{0}$-values drawn in Fig. 5 we would like to conclude that the cone surface ends somewhere in the interval $0.014<\mu_{0}<0.025$. Corresponding fits of the ellipse shape distorted by lattice artifacts can be done with an expression for the quark or pion mass obtained at next-to-leading order in lattice chiral perturbation theory, but still have a quite large uncertainty [11]. Therefore, we will not show them here.

\section{A first feasibility study at maximal twist}

So far we have not yet taken advantage of the expected $\mathscr{O}(a)$ improvement. For fixed $\beta$ and $\kappa=\kappa_{c}(\beta)$ one would like to change $\mu_{0}$ in order to vary the physical quark or pion mass. Since the statistical signals for the crossover turned out to be very noisy in this case, we instead decided to 


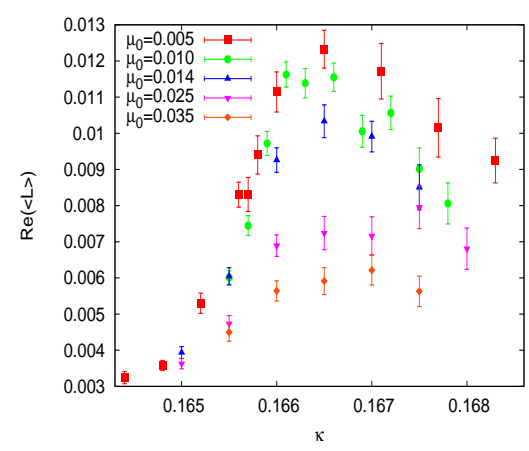

Figure 5: Polyakov loop scans as function of $\kappa$ at various $\mu_{0}$ for $\beta=3.75$.

fix $\mu_{0}$ and to vary $\beta$ and $\kappa=\kappa_{c}(\beta)$ accordingly. The values for $\kappa_{c}(\beta)$ can be estimated from the zero-temperature case (see e.g. $[6,7]^{1}$ ). For $\mu_{0}=0.040$ - which corresponds to a quite large pion mass value $m_{\pi} \simeq 1 \mathrm{GeV}$ - we have found the results shown in Figs. 6 . The 'critical' value $\beta_{t}=3.88$ can be translated into $T_{c} \simeq 280 \mathrm{MeV}$, which is again in the same ballpark in comparison with [20]. We conclude that this strategy to satisfy the requirements of an automatic $\mathscr{O}(a)$ improvement seems to work.
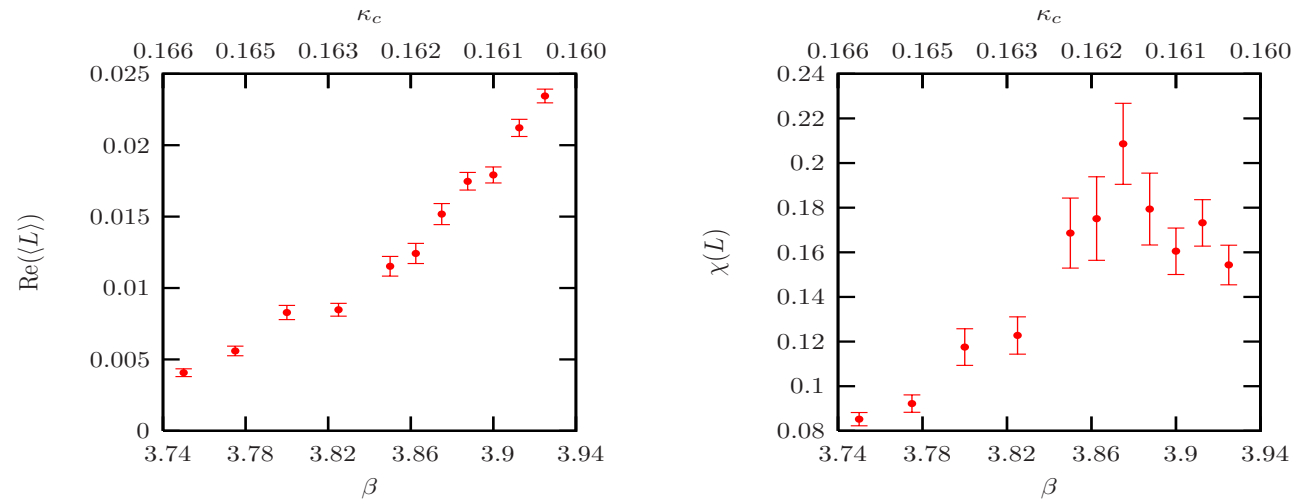

Figure 6: Polyakov loop (left) and its susceptibility (right) versus $\beta$ for maximal twist $\kappa=\kappa_{c}(\beta)$ at $\mu_{0}=$ 0.040 .

\section{Automatic $\mathscr{O}(a)$ improvement at $T \neq 0$}

Finally, we have checked that the automatic $\mathscr{O}(a)$ improvement really holds in the finitetemperature case. In the quenched approximation we have computed the pseudoscalar screening mass for varying spatial and temporal linear lattice sizes while keeping the physical mass ratio of pseudoscalar and vector states and the physical temperature fixed. The results are plotted in Fig. 7. They demonstrate nicely a linear behaviour in the square of the lattice spacing $a(\beta)$.

\section{Conclusions}

We are convinced that with the present study the necessary prerequisites for a serious non-zero

\footnotetext{
${ }^{1}$ We acknowledge the help of the ETM collaboration providing us also with data prior to publication.
} 


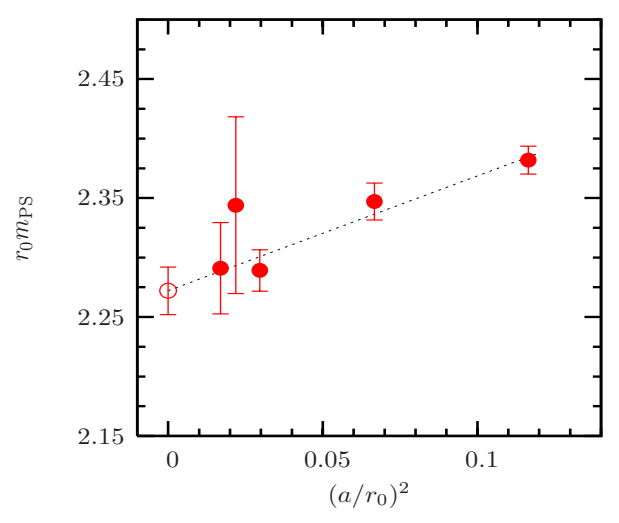

Figure 7: Pseudoscalar screening mass $m_{P S} r_{0}$ versus lattice spacing $\left(a / r_{0}\right)^{2}$ obtained for lattice sizes $N_{\sigma}=$ $24, \ldots, 32$ and $N_{\tau}=6, \ldots, 16$ at fixed $T / T_{c}=0.655(5)$ and $m_{P S} / m_{V} \simeq 0.75$.

temperature analysis with twisted-mass Wilson fermions have been collected in a sufficient manner. The structure of the three-dimensional phase diagram has been explored in the physical range for the two-flavour case. Still it is difficult to locate the (pseudo-) critical behaviour or crossover at fixed $\left(\beta, \kappa=\kappa_{c}(\beta)\right)$ along the direction of varying twisted-mass parameter $\mu_{0}$. Therefore, in a feasibility study, we have taken advantage of automatic $\mathscr{O}(a)$ improvement at fixed $\mu_{0}$ by passing through the crossover phenomenon changing $\beta$ and keeping close to the chiral critical line $\left(\beta, \kappa_{c}(\beta)\right)$ for which we can rely on twisted-mass results at zero temperature. For the quenched case we have demonstrated that $\mathscr{O}(a)$ improvement really works in the non-zero temperature setting. We are now in the position to start the determination of the critical temperature and of the equation of state with extrapolations to the limits of realistic light quark masses and to the continuum. In order to reach smaller pion masses we continue our investigation with $N_{\tau}=10,12$ on correspondingly larger spatial lattices.

\section{References}

[1] ALPHA Collaboration, R. Frezzotti, P. A. Grassi, S. Sint, and P. Weisz, JHEP 08 (2001) 058 , [hep-lat/0101001].

[2] R. Frezzotti and G. C. Rossi, JHEP 08 (2004) 007, [hep-lat/ 0306014 ].

[3] A. Shindler, Phys. Rept. 461 (2008) 37, [arXiv: 0707.4093 [hep-lat] ].

[4] K. Jansen, PoS (LAT2008) 010, [arXiv:0810.5634 [hep-lat]].

[5] M. Creutz, PoS (LAT2007) 007, [arXiv:0708.1295[hep-lat]].

[6] Ph. Boucaud et al. (ETM collaboration), Phys. Lett. B650 (2007) 304, [arXiv:hep-lat/0701012].

[7] Ph. Boucaud et al. (ETM collaboration), Comput. Phys. Commun. 179 (2008) 695, [arXiv:0803.0224 [hep-lat]].

[8] M. Creutz, [arXiv:hep-lat/9608024].

[9] M. Creutz, Phys. Rev. D76 (2007) 054501, [arXiv: 0706.1207 [hep-lat]]. 
[10] E.-M. Ilgenfritz et al. (tmfT collaboration), PoS (LAT2008) 206, [arXiv:0809.5228 [hep-lat]].

[11] E.-M. Ilgenfritz et al. (tmfT collaboration), Phys. Rev. D80 (2009) 094502, [arXiv:0905.3112 [hep-lat]].

[12] A. Ali Khan et al. (CP-PACS collaboration), Phys. Rev. D63 (2001) 034502, [arXiv: hep-lat/0008011].

[13] A. Ali Khan et al. (CP-PACS collaboration), Phys. Rev. D64 (2001) 074510, [arXiv:hep-lat/0103028].

[14] E.-M. Ilgenfritz, W. Kerler, M. Müller-Preussker, A. Sternbeck, and H. Stüben, Phys. Rev. D 69 (2004) 074511, [arXiv: hep-lat/0309057].

[15] E.-M. Ilgenfritz, W. Kerler, M. Müller-Preussker, A. Sternbeck, and H. Stüben, [arXiv:hep-lat/0511059].

[16] S. R. Sharpe and J. M. S. Wu, Phys. Rev. D70 (2004) 094029, [hep-lat/ 0407025 ].

[17] F. Farchioni et. al., Eur. Phys. J. C39 (2005) 421, [arXiv: hep-lat / 0406039 ].

[18] G. Münster, JHEP 09 (2004) 035, [arXiv : hep-lat / 0407006 ].

[19] F. Farchioni et. al., Eur. Phys. J. C42 (2005) 73, [arXiv : hep-lat/ 0410031 ].

[20] V. G. Bornyakov, et al. (DIK collaboration), [arXiv:0910.2392 [hep-lat]]. 